\title{
CORRECTION
}

\section{Correction to: BrdU/EdU dual labeling to determine the cell-cycle dynamics of defined cellular subpopulations}

\author{
Lachlan Harris $^{1} \cdot$ Oressia Zalucki $^{1} \cdot$ Michael Piper $^{1,2}$
}

Published online: 27 June 2018

○) Springer Nature B.V. 2018

\section{Correction to: \\ Journal of Molecular Histology (2018) 49:229-234 https://doi.org/10.1007/s10735-018-9761-8}

In the original publication of the article, two errors were made in describing the equations for $\mathrm{T}_{\mathrm{c}}$. The third equation should read:

$T_{\mathrm{c}}=\frac{T_{\mathrm{s}}}{\left(\text { BrdU }^{+v e} / \text { antibody }^{2+v e} \text { cells }\right)}$
Likewise, the final equation calculating $\mathrm{T}_{\mathrm{c}}$ for populations with a growth fraction of $<1$, should read:

$T_{\mathrm{c}}=\frac{T_{\mathrm{s}}}{\left(B r d U^{+v e} /\left(\text { antibody } y^{2+v e} \times G F\right)\right)}$

The authors apologise for the error and thank a reader for bringing this to their attention.

The original article can be found online at https://doi.org/10.1007/ s10735-018-9761-8.

Michael Piper

m.piper@uq.edu.au

Lachlan Harris

lachlan.harris@crick.ac.uk

Oressia Zalucki

o.zalucki1@uq.edu.au

1 The School of Biomedical Sciences, The Faculty of Medicine, The University of Queensland, Brisbane 4072, Australia

2 Queensland Brain Institute, The Faculty of Medicine, The University of Queensland, Brisbane, Australia 